Community members are active players, adding value to the lives of their $\mathrm{CN}$. By engaging citizens in the challenges and opportunities afforded by death, dying and loss, relinquishing control and sharing power, hospices introduce new actors into the end-of-life arena. There is evidence that it's scalable, flexible and adaptive.

\section{P-229 ABSTRACT WITHDRAWN}

\section{P-230 ARTIFICIAL INTELLIGENCE IN PALLIATIVE CARE: A SYSTEMATIC REVIEW TO IDENTIFY ITS SCOPE OF USE}

${ }^{1}$ Osamah Ahmad, ${ }^{2}$ Sarah Stanley, ${ }^{3}$ Stephen Mason, 4,5 ${ }^{6}$ Amara Nwosu. ${ }^{1}$ University of Liverpool, Liverpool, UK; ${ }^{2}$ Marie Curie Hospice Liverpool, Liverpool, UK; ${ }^{3}$ Palliative Care Unit, Liverpool, UK; ${ }^{4}$ Lancaster Medical School, Lancaster, UK; ${ }^{5}$ Marie Curie Hospice Liverpool, Liverpool, UK; ${ }^{6}$ Liverpool University Hospitals NHS Foundation Trust, Liverpool, UK

10.1136/spcare-2021-Hospice.244

Background New technologies, such as artificial intelligence (AI), supported by novel ways of linking and analysing data, are transforming the way that healthcare data is analysed. AI is increasingly being used to support healthcare delivery, and examples of palliative care application are emerging. AI is an umbrella term covering a variety of intertwined sub-concepts: machine learning refers to machine algorithms automatically improving themselves through experience, and neural networking refers to a form of this mimicking the way the human brain works. Deep learning is another form of machine learning, and natural language processing may refer to a variety of AI algorithms used to understand text intended for human recipients. However, the current scope of (and potential) use of AI in palliative care delivery has not been fully explored. The aim of this project was to define the scope of use of AI methodologies in palliative care studies.

Methods A systematic review of literature was conducted in accordance with the PRISMA guidelines. Four electronic databases were searched, in addition to grey literature searches. AI was used as an umbrella term to include keyword searches for the following: machine learning, deep learning, neutral networks and natural language processing.

Results Twenty-seven relevant articles were selected. The majority of studies described people with cancer $(n=10$, $37 \%)$, from general palliative $(n=8,30 \%)$ and intensive care populations $(n=4,15 \%)$. Studies using natural language processing were most common $(n=12,44 \%)$, with others mainly utilising machine learning $(n=10,37 \%)$, deep learning $(n=3$, $11 \%)$ and neural network $(n=2,8 \%)$ methodologies. A variety of outcomes were covered, with most studies predicting survival $(n=8,30 \%)$, identifying goals of care $(n=6,22 \%)$, analysing serious illness conversations $(n=2,9 \%)$ and reporting if palliative care best practice recommendations had been followed in clinical care $(n=2,9 \%)$.

Conclusion Most palliative care AI studies report cancer, use natural language processing and machine learning methods, to predict survival and analyse goals of care. Future studies need to explore how different AI methods can support palliative care, whilst carefully assessing the risks and limitations, to ensure effective use in the management of serious illness.

\section{P-231 THE QUALITY IMPROVEMENT METHODOLOGY USED TO CREATE DAFFODIL STANDARDS- TO IMPROVE END-OF- LIFE CARE}

${ }^{1}$ Catherine Millington-Sanders, ${ }^{2}$ Eve Barnes, ${ }^{3}$ Julie Pearce. ${ }^{1}$ Royal College of General Practitioners, London, UK; ${ }^{2}$ Great Western Hospitals NHS Foundation Trust, Swindon, UK; ${ }^{3}$ Marie Curie, London, UK

\subsection{6/spcare-2021-Hospice.245}

Background Most palliative care is provided by GPs and wider palliative care and community services (Mitchell, Loew, Millington-Sanders, et al., 2016). The Daffodil Standards (DS) were created in order to provide a free, accessible, evidencebased support around end-of-life care (see: https://www.rcgp. org.uk/clinical-and-research/resources/a-to-z-clinical-resources/ daffodil-standards/introduction.aspx). This work presents the quality improvement (QI) methodology used to establish an ongoing, national level process that helps engage practices in working to improve end-of-life care through QI and reflective practices.

Aims

- Supporting GP practices and Primary Care Networks, to provide high quality end-of-life care across their populations.

- Offering a structured approach - minimising variation in endof-life care experienced.

- Supporting GP teams - learning and development.

- Improving equity.

- Connecting GP care within compassionate community development (see: https://www.rcgp.org.uk/clinical-andresearch/resources/a-to-z-clinical-resources/daffodil-standards/ the-daffodil-standards/standard-8-general-practice-being-hubswithin-compassionate-communities.aspx).

Methods The QI methodology of Diagnose, Plan and Test, Implement and Embed, Sustain and Spread (NHS England, 2019) has been employed to evidence, establish and continually improve the Daffodil Standards.

Results The process:

Diagnose

- Review of learning from policy and research for gaps.

- Review of GP QI programmes/curriculum for scale service improvements to support general practice.

- Triangulation matrix between end-of-life care 'Ambitions', Istatements, GP qualitative research and feedback.

Plan and Test

- Development of draft DS headings.

- Consultation with end-of-life care partners.

- Reviewed from 9 to 8 core DS based on feedback.

- Organisational sign-off.

Implement and Embed

- Launch February 2019.

- Connected with England end-of-life care QOF 19/20.

Sustain and Spread (including developments)

- COVID-19 learning.

- Development of Older People's Care Home Standard.

- End-of-life care GP lead review from each nation to make more devolved nation accessible.

- Refresh of the Daffodil Standards.

- RCGP end-of-life care suite of webinars 2021.

- Planned evaluation 2021/22.

Conclusions This review of processes demonstrates how QI methodologies can be used on a national level to support 